\title{
LA TRADICIÓN EXPORTADORA DE LAS EMPRESAS HORTOFRUTÍCOLAS DE LA PROVINCIA DE ALICANTE
}

\author{
José Daniel Gómez López
}

\section{RESUMEN}

La tradición exportadora de la provincia de Alicante en el sector hortofrutícola se remonta al siglo XIX, sin embargo será a partir de la segunda mitad de la presente centuria cuando comenzará a tejerse una verdadera red empresarial especializada en la comercialización de variedades hortícolas. En este sentido, las actividades comerciales, especialmente externas, pasaron a representar uno de los principales ejes sobre el cual tanto empresas asociativas como privadas se apoyarían para integrarse en el mercado hortofrutícola mundial. Se trata de empresas que por regla general tienen una alta capacidad de gestión y elevada operatividad, atributos indispensables para poder competir frente a las grandes transnacionales fruteras en el Mercado Único Europeo.

\section{RESUMÉ}

La tradition pour l'éxportation de légumes et de fruites à la province d'Alicante date du XIXème siècle, mais ce n'est qu'à partir de la seconde moitié de la centaine actuelle quand il commencera la formation d'une vrai réseau spécialisée d'entrepreneurs pour le commerce des varietés des légumes. Alors, les activités commerciales, surtout externes, devinrent un des principals axes sur le quel sont appuyées les entreprises asociatives et privées pour l'intégration au marché mondiel de fruites et légumes. Il s'agit, en général, d'entreprises avec une haute capacité de gestion et d'opérativité, conditionnes nécesaires pour la concurrence au Marché Unique Europeèn front les grandes compagnies fruitières multinationelles.

La tradición exportadora de la provincia de Alicante en el sector hortofrutícola se remonta al siglo XIX, aunque su protagonismo en el contexto internacional se acrecentará a partir de la segunda mitad del siglo XIX. El Catálogo de Exportadores Españoles del año 1905, ya registraba entre las principales casas comerciales exportadoras de productos hortofrutícolas de España a un importante número de firmas con sede en la provincia de Alicante (vid. cuadro I), dedicadas fundamentalmente a la exportación de melón. Sin embargo será a partir de la segunda mitad de la presente centuria cuando comenzará a 


\begin{tabular}{|c|c|}
\hline Firma Comercial & Localidad \\
\hline Abad I. & Novelda \\
\hline Abad y Navarro & Novelda \\
\hline Aranda Chordi & Denia \\
\hline Aranda Morand, B. & Denia \\
\hline Arguimbau, J. & Denia \\
\hline Belda y Cía & Novelda \\
\hline Beresaluce, R. & Novelda \\
\hline Bermeti, M. & Denia \\
\hline Bordehore y Cía & Denia \\
\hline Cano, S. & Denia \\
\hline Cardona, J. R. & Denia \\
\hline Castelló y Cía & Novelda \\
\hline Cosmelli, Meyer y Cía & Denia \\
\hline Cuadrado, M. & Denia \\
\hline Devesa, M. & Denia \\
\hline Ferchen, J. & Denia \\
\hline Ivars y Cía & Denia \\
\hline Liand, J. & Denia \\
\hline Mahiques, Domenech & Denia \\
\hline Mayans, A. M. & Denia \\
\hline Martínez y Hernández & Novelda \\
\hline Merle, J. & Denia \\
\hline Miralles, D. & Denia \\
\hline Montón, B. & Denia \\
\hline Morales, V. & Denia \\
\hline Morand y Cía & Denia \\
\hline Navarro y Hno. & Novelda \\
\hline Nelson, T. & Denia \\
\hline Oliver y Cía & Denia \\
\hline Pallarés, C. & Denia \\
\hline Resoley, F. & Denia \\
\hline Riera, Vda. e H. & Denia \\
\hline Rogers y Cía & Denia \\
\hline Sala Beresaluce & Novelda \\
\hline Seller Hnos. & Denia \\
\hline Soller, E. & Denia \\
\hline Trenor y Cía & Denia \\
\hline Villalta, $\mathrm{T}$. & Denia \\
\hline Viaplana, S. & Denia \\
\hline TOTAL ALICANTE & 39 \\
\hline TOTAL VALENCIA & 31 \\
\hline TOTAL OTROS & 5 (incl. Murcia) \\
\hline TOTAL ESPAÑA & 75 \\
\hline
\end{tabular}

FuENTE: MINISTERIO DE ESTADO, Catálogo de exportadores españoles, 1905. Elaboración propia. 
tejerse una verdadera red empresarial especializada tanto en la producción como en la comercialización de variedades hortícolas.

\section{Origen y expansión del sector empresarial}

El inicio de las actividades empresariales hortofrutícolas de carácter internacional, está íntimamente asociado a la expansión del cultivo de tomate en la provincia, a partir del año 1940. Se trataba de empresas cosechero-exportadoras familiares de origen canario como Bonny, dedicadas a la producción y comercialización de tomate en el Archipiélago desde los años treinta de la presente centuria. En Alicante se instala y desarrolla una organización productivo-comercial análoga a la existente en Canarias. Se trata de empresas que arriendan o compran fincas ejecutando en ellas labores de infraestructura tales como construcción de pozos (posteriormente redes conductoras de aguas, embalses), vías de comunicación y almacenes para empaquetado. El sistema de explotación seguido por estas empresas contemplaba en algunos casos la figura del asalariado, y en otros el de medianero, ya que el interés de estas firmas radicaba esencialmente en los procesos de comercialización (RODRÍGUEZ BRITO, W., 1986, 357).

En este contexto, también se introducen en la actividad productivo-comercial provincial, casas comerciales de origen valenciano tradicionalmente dedicadas a labores de comercialización tanto interna como externa (intermediación). Casa Mitjana Hermanos S.L., se establece en Canarias en los años treinta de la presente centuria, con almacén de empaquetado propio. Durante la Guerra Civil sus actividades cesan momentáneamente, sin embargo finalizada la contienda, vuelve a erigirse como una de las principales empresas dedicadas a la compra de fruta en muelle de origen para su envío y distribución en la Península. Después de un fulgurante desarrollo en el período 1953-1970, su actividad en el Archipiélago comienza a decrecer (RODRÍGUEZ BRITO, W., 1986, 199).

Casamitjana S.A. surge en el municipio de Mutxamel (Alicante) en los años cincuenta, dedicándose además de las labores comerciales a las productivas, centrándose principalmente en el cultivo de tomate que hasta entonces recibía de Canarias. Además del tomate que adquiere de los cosecheros individuales, esta empresa también lo cosecha en sus propias fincas instaladas en Sant Vicent del Raspeig, Novelda, Fortuna (Murcia) y la provincia de Almería (GINER GONZÁLEZ, M., 1981, 199). En el año 1988 figuraba en el Catálogo de Exportadores Españoles con una exportación de tomate cuyo valor se aproximaba a los 153 millones de pesetas, un 43 por ciento menos que en la anterior campaña. En 1991 esta firma había desaparecido del Catálogo de Exportadores, presumiblemente debido al cese de actividades.

En las postrimerías de la década de los cincuenta comienzan a aparecer en Alicante empresas privadas que muy pronto ocuparán un lugar destacado en el sector exportador español. Exportadores de Tomate de Alicante S.A. (ETASA), se constituye en el mes de marzo de 1959, por fusión de diversas empresas dedicadas a la producción y exportación de tomate fresco de invierno; de este modo se trataba de alcanzar una dimensión óptima de producción así como un tamaño de empresa adecuado para competir por mayores cuotas de mercado.

Inicialmente sus explotaciones se extendieron por todo el Campo de Alicante y Águilas (Murcia), instalándose posteriormente en la Islas Canarias, donde pasa de ocupar el decimoséptimo lugar entre los exportadores de Tenerife en el período 1962-63, al primer lugar en la campaña 1965-66 con 2.566.590 kilogramos. Entre los términos de Alicante y Jijona la empresa ETASA poseía en propiedad una superficie de 924 hectáreas, donde disponía de 
unos caudales de agua para riego próximos a los 215 litros por segundo, traídos de Castalla y Onil. Para regular el riego contaba asimismo con varios embalses en sus propiedades, que sumaban una capacidad de 200.000 metros cúbicos (Valencia Fruits, n. ${ }^{\circ}$ 642, 1974.

El empaquetado de hortalizas se realizaba en la Central Hortofrutícola instalada en el término municipal de San Juan, con una capacidad de confección de 20.000 ceretos diarios de tomate fresco (100.000 kilogramos), y 4.000 cajas de apio de 12-14 kilogramos de peso. Además de estas variedades hortícolas la empresa también producía y exportaba guisantes, bróculi, maíz dulce y espárragos, desarrollando estas producciones tanto en la provincia de Alicante como en la finca de 120 hectáreas que poseía en el Prat de Llobregat (Barcelona). En el año 1975 para confeccionar su producción inauguró una Central Hortofrutícola en Vila de Camps (Bajo Llobregat), que ocupaba una superficie de 4.000 metros cuadrados.

Además de las labores productivas propias, la empresa ETASA creó en el año 1973 una firma en sociedad con la empresa francesa Compagnie Fruitière (Marseille) y Bonny S.A., de Alicante, para la producción en Águilas (Murcia) de variedades hortícolas bajo abrigo plástico, además de cultivar endivia al aire libre para exportar principalmente a Francia, Holanda y Bélgica. Asimismo por esta época (1974), ETASA formaba parte de un reducido número de empresas españolas (nueve) autorizadas por el Ministerio de Agricultura para poseer viveros de agrios, teniendo asignado un cupo de 500.000 plantones anuales. Esta actividad venía a completar los viveros de almendros y barbados de vid (30 hectáreas) que la empresa ya poseía (Valencia Fruits, n. ${ }^{\circ} 642,1974$ ).

Para planta ornamental de interior y exterior, la empresa ETASA contaba con 42 invernaderos (14 con calefacción) y un Garden Center instalado en el anexo a la Central Hortofrutícola de San Juan, donde se comercializaba todo tipo de planta. Esta actividad de ETASA llegó a tener tanta importancia que en el año 1974, con motivo de la celebración en la ciudad de Valencia del certamen Iberflora, fue la única empresa exportadora representante de la provincia de Alicante que se dedicaba a la comercialización de Planta de Interior, Exterior, Árboles y Arbustos, Jardinería, Frutales y Cítricos (viveros) (Iberflora, 1974).

Hasta el año 1973 la empresa Exportadora de Tomate de Alicante, S.A. (ETASA), ocupaba el primer lugar entre los exportadores peninsulares. Su producción de tomate de invierno oscilaba entre las 15.000 y 16.000 toneladas, de las que enviaba hacia el exterior un 60 por ciento. Para comercializar en los mercados externos se creó la filial ETASA (U.K.) Ltd., situada en el Reino Unido, cuyo volumen de ventas en el año 1974 ascendió a 2,5 millones de libras (Valencia Fruits, 642, 1974). En la segunda mitad de los años ochenta cesan las actividades de esta empresa, principalmente a causa de una gestión deficiente.

El cese de actividades de numerosas empresas alicantinas ha ido mermando la capacidad exportadora hortícola provincial tanto en el contexto del Sureste peninsular como nacional (vid. cuadro II). La supresión de los cupos para la exportación de tomate supuso un duro golpe para este sector en beneficio de otras empresas radicadas fuera de la provincia. Asimismo la incorporación de nuevas técnicas en los cultivos y la expansión de los invernaderos en Almería y Murcia, han restado protagonismo a la producción tanto de tomate como posteriormente de pimiento alicantino.

Tomando como referencia un estudio realizado en el año 1966 (NAVARRO SALA, J.A., 1966, 155), y sirviéndonos de los censos de las últimas campañas de exportación realizados por la Asociación de Cosecheros-Exportadores de Tomate de Invierno de la provincia de Alicante (cuadro III), se aprecia que hasta la campaña 1987-88, existe un crecimiento sostenido tanto de los envíos de tomate como de la capacidad media de las empresas. Sin embargo inmediatamente después estos índices se reducen sensiblemente, 
EVOLUCIÓN DE LAS EXPORTACIONES EMPRESARIALES HORTÍCOLAS DE ALICANTE, ALMERÍA Y MURCIA (en millones de pesetas)

\begin{tabular}{|lrrrrr|}
\hline Producto & $\mathbf{1 9 8 3}$ & $\mathbf{1 9 8 5}$ & $\mathbf{1 9 8 6}$ & $\mathbf{1 9 8 8}$ & $\mathbf{1 9 9 0}$ \\
\hline Tomates 1/11-14/05 & & & & & \\
Alicante & 2.519 & 2.900 & 3.009 & 3.104 & 3.214 \\
Almería & 220 & 290 & 474 & 422 & 664 \\
Murcia & 3.584 & 3.743 & 2.848 & 5.828 & 5.139 \\
TOTAL PENÍNSULA & 10.174 & 10.878 & 10.586 & 14.203 & 10.030 \\
\hline Tomates 15/05-30/09 & & & & & \\
Alicante & 101 & 182 & 191 & 70 & - \\
Almería & - & 59 & 126 & 111 & - \\
Murcia & 22 & 16 & 224 & $\mathrm{~s} . \mathrm{d}$. & - \\
TOTAL PENÍNSULA & 219 & 511 & 972 & 429 & - \\
\hline Pimientos & & & & & \\
Alicante & 1.090 & 1.965 & 2.175 & 2.538 & 2.925 \\
Almería & 960 & 1.461 & 3.511 & 4.269 & 7.057 \\
Murcia & 404 & 1.192 & 945 & 1.940 & 1.483 \\
TOTAL ESPAÑA & 4.760 & 7.840 & 10.877 & 19.162 & 18.506 \\
\hline
\end{tabular}

Fuente: SECRETARÍA DE ESTADO DE COMERCIO, Censo oficial de exportadores. Elaboración propia.

CUADRO III

EVOLUCIÓN DE LAS EXPORTACIONES EMPRESARIALES DE TOMATE EN LA PROVINCIA DE ALICANTE

\begin{tabular}{|ccccc|}
\hline & $\begin{array}{c}\text { Total exportado } \\
\text { por campaña } \\
\text { Campaña }\end{array}$ & $\begin{array}{c}\text { Número de } \\
\text { (1) }\end{array}$ & \multicolumn{2}{c|}{$\begin{array}{c}\text { Media de exportación } \\
\text { por empresario }\end{array}$} \\
\hline $1985-86$ & 57.949 .854 & 57 & 1.016 .664 & 169.444 \\
$1986-87$ & 56.586 .300 & 47 & 1.203 .964 & 200.661 \\
$1987-88$ & 59.054 .466 & 46 & 1.283 .793 & 213.965 \\
$1988-89$ & 56.251 .422 & 48 & 1.171 .905 & 195.317 \\
$1989-90$ & 46.868 .688 & 38 & 1.233 .387 & 205.564 \\
$1990-91$ & 40.439 .970 & 32 & 1.263 .749 & 210.625 \\
\hline TOTAL & 317.150 .690 & & 1.183 .398 & 197.233 \\
\hline
\end{tabular}

FUENTE: ASOCIACIÓN COSECHEROS - EXPORTADORES DE TOMATE DE INVIERNO DE LA PROVINCIA DE ALICANTE. Elaboración propia.

Obs.: (1) En Kilogramos.

(2) En bultos de 6 kilogramos netos. 
hasta que en la campaña 1990-91 la capacidad media de exportación de las empresas vuelve a experimentar un ligero crecimiento debido principalmente a la reducción de su número total. En este sentido en el último quinquenio, la exportación total de tomate ha experimentado una reducción del 30 por ciento (vid. cuadro III).

Tomando como base la campaña 1985-86 y considerando a las empresas que exportaron en esa campaña como censo inicial de análisis, la estructura durante las seis campañas siguientes revela, a grandes rasgos, la extremada movilidad de este sector, puesto que tan sólo el 22,8 por ciento de las empresas han mantenido una línea de continuidad en su actividad comercial externa durante las seis campañas analizadas, mientras que el 77,2 por ciento restante se trata de empresas que realizaron envíos esporádicos, dada la escasa operatividad de las mismas (capacidad económica y escasa dimensión — vid. cuadro IV) .

La limitada capacidad comercial de la mayor parte de las empresas exportadoras de tomate de invierno (vid. cuadro V), configura una situación de extrema fragilidad, princi-

CUADRO IV

EVOLUCIÓN DE LA ESTRUCTURA EXPORTADORA EMPRESARIAL DEL TOMATE EN LA PROVINCIA DE ALICANTE

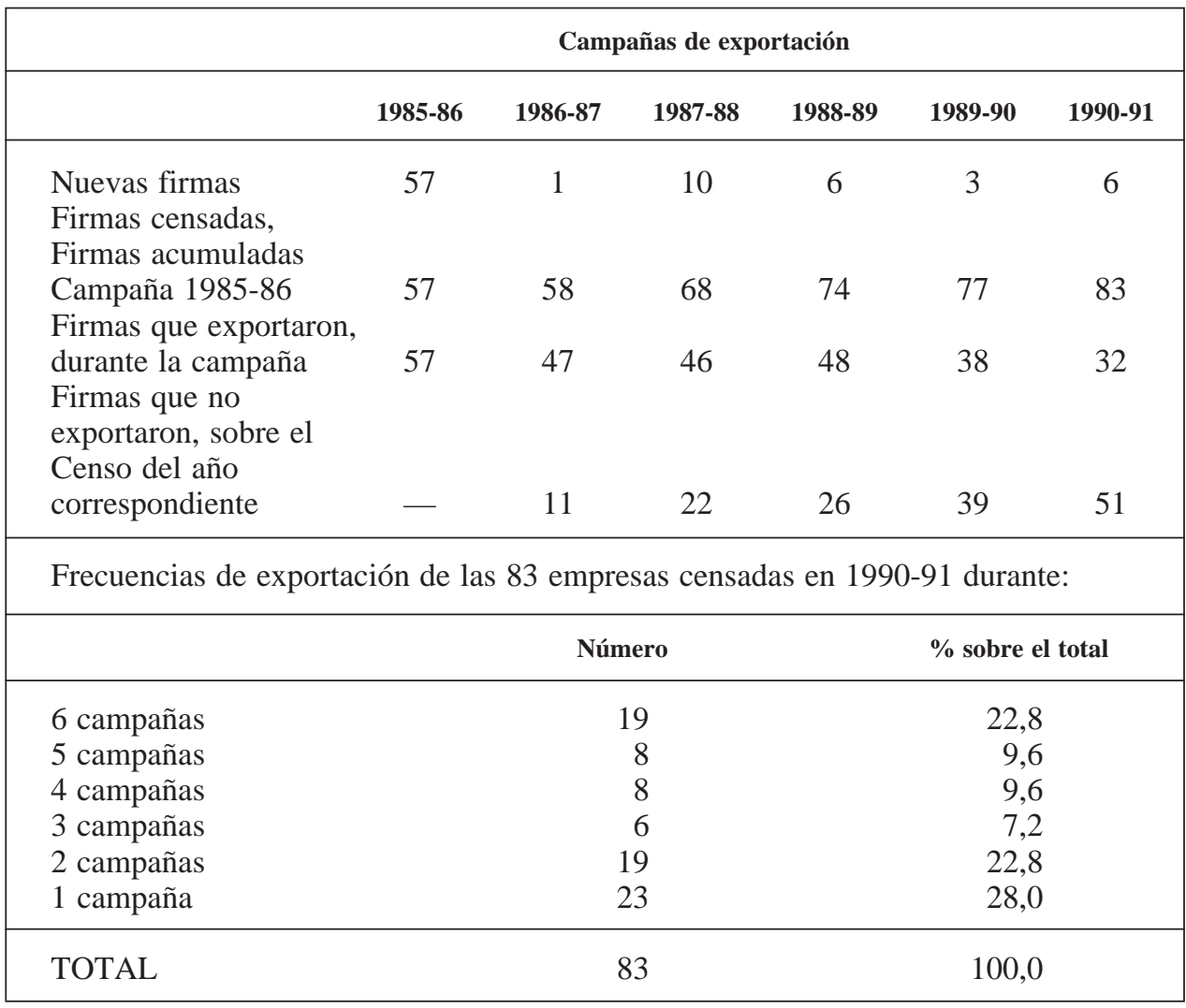

FUENTE: ASOCIACIÓN COSECHEROS - EXPORTADORES DE TOMATE DE INVIERNO DE LA PROVINCIA DE ALICANTE. Elaboración propia. 
EVOLUCIÓN DE LA CAPACIDAD COMERCIAL RELATIVA DE LAS EMPRESAS EXPORTADORAS DE TOMATE EN RELACIÓN A LA MEDIA (EN PORCENTAJE)

\begin{tabular}{|lcccccc|}
\hline \multicolumn{7}{c|}{ Campañas de exportación } \\
\hline & $\mathbf{1 9 8 5 - 8 6}$ & $\mathbf{1 9 8 6 - 8 7}$ & $\mathbf{1 9 8 7 - 8 8}$ & $\mathbf{1 9 8 8 - 8 9}$ & $\mathbf{1 9 8 9 - 9 0}$ & $\mathbf{1 9 9 0 - 9 1}$ \\
\hline $\begin{array}{l}\text { Empresas que } \\
\text { exportaron más } \\
\text { que la media } \\
\begin{array}{l}\text { Empresas que } \\
\text { exportaron menos } \\
\text { que la media }\end{array}\end{array}$ & 31,6 & 34,0 & 37,0 & 39,5 & 34,2 & 31,2 \\
\hline TOTAL FIRMAS & 100,0 & 100,0 & 100,0 & 100,0 & 100,0 & 100,0 \\
\hline
\end{tabular}

FUENTE: ASOCIACIÓN COSECHEROS - EXPORTADORES DE TOMATE DE INVIERNO DE LA PROVINCIA DE ALICANTE. Elaboración propia.

\section{CUADRO VI}

EVOLUCIÓN DE LA CAPACIDAD EXPORTADORA DE LAS EMPRESAS DE TOMATE DE INVIERNO DE LA PROVINCIA DE ALICANTE (EN PORCENTAJE)

\begin{tabular}{|lrrrrr|}
\hline Campañas & $\begin{array}{c}\text { Menos de 500 } \\
\text { toneladas }\end{array}$ & $\begin{array}{c}\mathbf{5 0 0 - 1 . 0 0 0} \\
\text { toneladas }\end{array}$ & $\begin{array}{c}\mathbf{1 . 0 0 1 - 5 . 0 0 0} \\
\text { toneladas }\end{array}$ & $\begin{array}{c}\text { Más de 5.000 } \\
\text { toneladas }\end{array}$ & TOTAL \\
\hline 1985-86 & & & & & \\
Empresas & 49,0 & 19,5 & 28,0 & 3,5 & 100,0 \\
Total exportado & 7,0 & 13,5 & 53,0 & 26,5 & 100,0 \\
$\begin{array}{l}\text { 1987-88 } \\
\text { Empresas }\end{array}$ & 43,5 & 13,0 & 39,0 & 4,5 & 100,0 \\
Total exportado & 5,5 & 7,0 & 56,0 & 31,5 & 100,0 \\
1989-90 & & & & & \\
Empresas & 38,5 & 24,0 & 32,0 & 5,5 & 100,0 \\
Total exportado & 4,5 & 14,0 & 43,0 & 39,0 & 100,0 \\
1990-91 & 40,5 & 12,5 & 44,0 & 3,0 & 100,0 \\
Empresas & 5,5 & 7,5 & 62,0 & 25,0 & 100,0 \\
Total exportado & & & & & \\
\hline
\end{tabular}

FUENTE: ASOCIACIÓN COSECHEROS - EXPORTADORES DE TOMATE DE INVIERNO DE LA PROVINCIA DE ALICANTE. Elaboración propia.

palmente si se considera que la actual estrategia comercial parte de la necesidad de potenciar estructuras empresariales, tanto privadas como asociativas, a través del aumento de su tamaño y del fortalecimiento del sector. Aunque en líneas generales se observa un aumento potencial del tamaño medio de las empresas (vid. cuadro VI), las pequeñas empresas en la última campaña (1990-91) sobrepasaban el 50 por ciento del censo, concentrando éstas empresas tan sólo el 13 por ciento del volumen total exportado por la Asocia- 
ción de Cosecheros-Exportadores de Alicante.

En el sector de productos hortícolas cultivados bajo abrigo plástico, en la provincia de Alicante destaca la empresa cooperativa Surinver, Soc. Coop., instalada en el término municipal de El Pilar de la Horadada, y en la actualidad una de las cooperativas más importantes de la Comunidad Valenciana y de España.

\section{Grupo sindical de colonización «Horadada»}

Los Grupos Sindicales de Colonización nacen en España al amparo de la Ley de Colonizaciones de Interés Local del año 1940, como respuesta a la necesidad de dar vida a agrupaciones de agricultores que perseguían finalidades concretas, con una organización sencilla y flexible y unos requisitos mucho menos exigentes que los de las cooperativas. De ahí su éxito inicial.

Su personalidad jurídica fue reconocida por la Ley de Ordenación Rural de 1968 y por la Sindical de 17 de febrero de 1971. La Ley de Reforma y Desarrollo Agrario de 1973 vino a confirmar la existencia y capacidad de tales Grupos. El Grupo Sindical de Colonización «Horadada n. ${ }^{\circ}$ 14773», se crea al amparo de esta legislación en el año 1973, agrupando inicialmente 15 agricultores de la entonces pedanía de El Pilar de la Horadada (municipio de Orihuela). Su objetivo principal era la comercialización en común de sus producciones, lo que les permitiría asegurar su propio margen de venta y el valor añadido generado en el proceso comercial.

La alcachofa y el pimiento constituyeron los primeros productos hortícolas comercializados; el pimiento por aquel entonces ya se cultivaba bajo abrigo plástico. Posteriormente, a partir de la segunda campaña (1975-76), el tomate pasa a engrosar el cuadro de producciones hortícolas del Grupo de Colonización, si bien la comercialización de este producto nunca fue excesivamente importante.

El éxito logrado en los primeros años de actuación, estimuló el ingreso de nuevos socios y, por extensión, aumentó sustancialmente el volumen de los productos aportados para la comercialización en común. Esta concentración de la oferta y el fortalecimiento de la estructura de gestión del Grupo, supuso su reconocimiento en el año 1975 como Agrupación de Productores Agrarios. En efecto, por la Orden del Ministerio de Agricultura, Pesca y Alimentación, de fecha 6 de marzo de 1975 (B.O.E. del 15 de abril), el Grupo Sindical de Colonización «Horadada n. ${ }^{\circ}$ 14.773» fue calificado como A.P.A. n. ${ }^{\circ} 14$.

Las Agrupaciones de Productores Agrarios fueron creadas en España por la Ley 29/72 de 22 de julio. La calificación como APA, para cualquier entidad asociativa (Grupo Sindical de Colonización, Cooperativa), suponía en cuanto a beneficios, subvenciones de 3,2 y 1 por ciento, durante el primer, segundo y tercer año respectivamente del valor de la producción comercializada por la entidad; también créditos oficiales hasta un máximo del 70 por ciento del valor base de los productos entregados a la entidad, para proporcionar anticipos contra entrega de productos, así como los beneficios de zona de Preferente Localización Industrial Agraria, a las instalaciones necesarias para almacenamiento, tipificación y acondicionamiento de los productos. Estas ayudas se concretaban principalmente en subvenciones de hasta el 20 por ciento de la inversión real, y créditos oficiales para el 80 por ciento del resto de la inversión.

Por otro lado los requisitos mínimos a cumplir se concretaban en aportar a la Agrupación la totalidad de los productos para cuya comercialización había sido calificada, constitución de un fondo de reserva especial, volúmenes mínimos de comercialización y dispo- 
ner de una gerencia cualificada en cuestiones comerciales. En ocasiones las subvenciones y beneficios previstos en la Ley se vieron recortados por insuficiente dotación presupuestaria. Asimismo eran constantes las denuncias por insuficiente agilidad en la concesión de créditos de campaña destinados a que las entidades pudieran dar anticipos a los socios contra entrega de sus productos (ÁLVAREZ GÓMEZ, J., 1984, 85).

\section{II.1. Sociedad agraria de transformación «Horadada $n .^{\circ} 2.348 »$}

Al amparo de lo establecido en el Real Decreto 1.776/1981 de 3 de agosto, y con sujección a sus normas, se constituye la Sociedad Agraria de Transformación que se denominará Sociedad Agraria de Transformación Horadada n. ${ }^{\circ}$ 2.348, inscrita en el Registro General de S.A.T. del Instituto de Relaciones Agrarias del Ministerio de Agricultura, Pesca y Alimentación con fecha 26 de septiembre de 1982.

La transformación de Grupo Sindical de Colonización a Sociedad Agraria de Transformación no afectó sustancialmente el régimen fiscal por el que hasta ese momento se regían los Grupos Sindicales. Así según el Real Decreto 1776/81, «las SAT continuarán disfrutando de las exenciones fiscales y beneficios de cualquier clase actualmente reconocidos». Entre los principios que regían el estatuto de la SAT Horadada, destaca el artículo 2, referente al Capital Social, donde se especifica que «el capital social de la SAT está constituido por las participaciones realizadas por los socios de la SAT, para este fin». Las participaciones se hacían de acuerdo a la superficie agrícola de los socios. Así por cada tahúlla destinada a productos cultivados al aire libre, el socio tendría una participación, y tres participaciones y media por cada tahúlla de productos cultivados en invernadero, especificando «que las participaciones servirían de base para la participación de los beneficios sociales comunes que se obtengan».

Como Sociedad Agraria de Transformación, el volumen de transacciones comerciales se vio incrementado extraordinariamente. Además del aumento de la oferta, las actividades exportadoras pasaron a constituir la columna vertebral de la Sociedad, incrementando los mercados de destino principalmente hacia los países de la Comunidad Económica Europea. Esta dinámica comercial estimuló no solamente el ingreso de nuevos socios (86 en el año 1974; 252 en 1977) (COSTA MAS, J.; CANALES, G., 1980, 188), sino que por efecto inducido, propició la constitución en la región de otras SATs, para la comercialización en común.

\section{Surinver, Sociedad Cooperativa}

Al amparo de lo establecido en la Disposición Adicional Tercera de la Ley 3/1987 de 2 de abril General de Cooperativas se transforma en Sociedad Cooperativa Agraria la anteriormente denominada «Sociedad Agraria de Transformación Horadada n. ${ }^{\circ}$ 2.348» (Estatutos de la Cooperativa Agrícola Surinver, Soc. Coop.). Esta transformación se produce especialmente, a partir de los cambios que en materia fiscal introduce la Ley sobre Régimen Fiscal de las Cooperativas (Boletín Oficial de las Cortes de 2 de marzo de 1989).

Mientras los Estatutos de la SAT Horadada contemplaban básicamente como objeto social, en su artículo 1 (Art. 1), la comercialización en común de las producciones obtenidas por los socios, bajo el régimen estatutario de Cooperativa y como Organización de Productores de Frutas y Hortalizas (O.P.F.H) «el objeto de la sociedad es la prestación de suministros y servicios, y la realización de operaciones encaminadas al mejoramiento económico y técnico de las explotaciones de los socios» (Art. 2). 
La introducción de los términos «económicos» y «técnico», son extraordinariamente novedosos, por cuanto amplían los objetivos de la Cooperativa Surinver, cuya labor fue reconocida por el Ministerio de Agricultura, Pesca y Alimentación, al concederle en el año 1991 una placa al Mérito Agrícola por el «saber hacer» de esta Cooperativa, considerada la primera de la provincia de Alicante y una de las más importantes de España (Valencia Fruits, 23-04-1991).

\section{III.1. Base social e infraestructura productiva}

El espacio sobre el cual se extiende la Cooperativa Surinver «son las provincias de Alicante y Murcia» (Estatutos de Surinver, Soc. Coop.). Es importante destacar que mientras en los estatutos de la SAT Horadada aparecía claramente delimitado el ámbito geográfico de la misma (en Alicante: pedanía de El Pilar de la Horadada, Torrevieja, San Miguel de Salinas y Los Montesinos. En Murcia: San Pedro del Pinatar, San Javier, pedanía de Gea y Truyols y Dolores de Pacheco), en los nuevos estatutos esta demarcación no solamente desaparece sino que se ajusta a una nueva dinámica espacial fuertemente interrelacionada por nexos de carácter económico-social y sustentada en función de las infraestructuras viales.

Por otro lado también es verdad que el espacio asume paulatinamente nuevas formas organizativas, como consecuencia de la articulación que en torno a las microeconomías regionales se van tejiendo. De este modo, la progresiva aparición de estructuras asociativas y privadas en la comarca Meridional de la provincia de Alicante y Campo de Cartagena en la provincia de Murcia, si bien evidencian la progresiva internacionalización de las actividades agrícolas regionales (comercialización), por otro lado limitan la movilidad de los elementos que participan en el proceso productivo. En este sentido, mientras en el año 1980 los socios de la actual Cooperativa Surinver, pertenecían a 18 localidades diferentes, una década más tarde éstos se circunscriben a 8 localidades (vid. cuadro VII).

La superficie de producción nucleada en torno a la Cooperativa Surinver, se ha incrementado sustancialmente en el transcurso de las últimas campañas (vid. cuadro VIII). Es importante destacar que el cultivo protegido apenas ha experimentado variaciones en los últimos años, lo que se debe, según informaciones recogidas por el autor, al elevado costo de construcción de nuevos invernaderos así como a la ausencia de créditos a bajo interés. El costo medio del metro cuadrado de invernadero en 1990-91 ascendía a 1.500 pesetas, de las que el IRYDA concedía el 30 por ciento de la financiación a fondo perdido, pero tan sólo cuando el proyecto estuviese concluido, lo que en muchos casos era técnicamente imposible al no contar el agricultor con los suficientes ahorros(*). Aunque se trata de una figura productiva en claro proceso de desaparición (principalmente en los cultivos intensivos bajo plástico) en 1990 figuraban en el cuadro de asociados a la Cooperativa un total de 110 medieros.

Como ocurre en el Campo de Dalías (Almería), Campo de Cartagena, Águilas y Mazarrón (Murcia), en El Pilar de la Horadada existen serios problemas para la contratación de mano de obra, principalmente cuando ésta es de marcada tendencia estacional. En este sentido es práctica habitual entre los propietarios de los invernaderos recurrir a la contratación de mano de obra extranjera (norteafricana), principalmente en períodos de cosecha, ya que se trata de productos perecederos (hortalizas y flores) que necesitan ser

(*) Agradecemos la inestimable colaboración de los Sres. Adolfo García y José Garcerán por la información y ayuda prestada. 
EVOLUCIÓN DEL NÚMERO DE ASOCIADOS DE LA COOPERATIVA SURINVER (ALICANTE), POR LOCALIDADES. EN NÚMEROS ABSOLUTOS

\begin{tabular}{|llrr|}
\hline Localidad & Provincia & 1980 (1) & $\mathbf{1 9 9 0}$ \\
\hline El Pilar de la Horadada & Alicante & 164 & 268 \\
Torre de la Horadada & Alicante & 2 & - \\
San Pedro del Pinatar & Murcia & 51 & 81 \\
San Javier & Murcia & 36 & - \\
El Mirador & Murcia & 3 & - \\
Los Alcázares & Murcia & 1 & - \\
Torre Pacheco & Murcia & 2 & 2 \\
Balsicas & Murcia & 3 & 3 \\
Sucina & Murcia & 5 & - \\
Murcia & Murcia & 4 & - \\
Torrevieja & Alicante & 1 & - \\
Orihuela & Alicante & 1 & - \\
Elche & Alicante & 1 & - \\
Callosa de Segura & Alicante & 1 & - \\
Los Montesinos & Alicante & 1 & - \\
Cartagena & Murcia & 1 & \\
Alhama de Murcia & Murcia & 1 & - \\
Roquetas de Mar & Almería & - & - \\
Santiago de la Ribera & Murcia & 400 & - \\
\hline TOTAL & 279 & & - \\
\hline
\end{tabular}

Fuente: Para 1980, COSTA MAS, J. - CANALES MARTínEZ, G.: «El cultivo de invernadero y la comercialización agraria en Orihuela y Campo de Cartagena», Cuadernos de Geografía, núm. 27, Valencia, 1980. Para 1990, SURINVER, SOC. COOP. Elaboración propia.

CUADRO VIII

EVOLUCIÓN DEL CUADRO ASOCIATIVO Y ESTRUCTURAL DE LA COOPERATIVA SURINVER (ALICANTE)

\begin{tabular}{|ccc|}
\hline Campañas & Número de socios & Superficie \\
\hline $1986-87$ & 352 & 1.343 \\
$1987-88$ & 371 & 1.376 \\
$1988-89$ & 379 & 1.463 \\
$1989-90$ & 384 & 1.545 \\
\hline
\end{tabular}

FUENTE: SURINVER, SOC.COOP.

recogidos rápidamente al depender su precio de la calidad que presente el producto. No existen datos que cuantifiquen en su justa medida la incidencia de este colectivo en la región. Sin embargo en el año 1989 con motivo de la firma del convenio colectivo del campo en la provincia de Murcia, se calculó que del total de trabajadores existentes (55.000), el 27 por ciento estaba integrado por andaluces, extremeños y marroquíes.

En la Cooperativa trabaja un total de 40 personas fijas (oficinas y encargados de área), mientras que los trabajadores estacionales (almacenes de manipulado) ascienden en plena campaña de producción a 600 . 


\section{III.2. Gestión comercial}

Los productos una vez desembarcados en el muelle del almacén de manipulado o área de recepción, pasan por un control mediante el cual se conoce tanto la cantidad y peso aportado por cada socio como la calidad del producto (aspecto físico y calibre). La confección y expedición del fruto constituye el último tramo de la cadena realizada por el almacén de manipulado.

Una vez introducida la carga en el camión frigorífico, ésta accede al mercado receptor (distribución) mediante contrato o libremente. Sin embargo la comercialización generalmente se realiza bajo fórmulas contractuales (compra en firme). Entre la Cooperativa y el mayorista de destino (importador-distribuidor) existe una relación de mutua confianza, lograda a través de una larga actuación comercial. En este sentido la Cooperativa se compromete a atender los pedidos solicitados por el mayorista aún a sabiendas de que su propia producción no es suficiente (en este caso se recurre a contratar productos agrícolas de terceros). Por otro lado el mayorista asume la responsabilidad (riesgo) de adquirir todo el producto enviado desde el centro de producción, aún cuando esto represente serios inconvenientes (pérdida de beneficio).

La alcachofa y el pimiento fueron las primeras variedades hortícolas comercializadas por la Cooperativa Surinver; la alcachofa se enviaba mayoritariamente al mercado francés. Posteriormente se amplió la gama de variedades ofertadas tanto hortícolas como frutícolas y florales, aunque en la actualidad continúa siendo el pimiento la variedad más comercializada (vid. cuadro IX).

Tratándose de una Cooperativa fuertemente integrada en la esfera del comercio hortofrutícola internacional, su producción se destina fundamentalmente hacia los mercados externos (vid. figura 1), representando éstos el 68 por ciento del volumen total comercializado en la última campaña (1990-91). Es importante destacar que los envíos hacia el mercado interior, en la últimas campañas mantienen cuotas muy equilibradas (32 por ciento tanto en la campaña 1986-87 como en 1990-91).

Los principales mercados a los que se dirigen los envíos de la Cooperativa, están situados en los países del entorno comunitario europeo (vid. figura 2 y cuadro X), aunque según la variedad hortícola exportada, la primacía de uno u otro país puede variar sensiblemente. Así el principal cliente del pimiento en la campaña 1990-91 es Francia (52\%), seguida de Alemania (39\%) y Austria (3\%). Sin embargo las lechugas eran absorvidas principalmente por Alemania (29\%), Reino Unido (22\%) y los Países Bajos (13\%). En cuanto a la comercialización interna, en la campaña 1990-91 se dirigía principalmente a Madrid (32\%), Barcelona (29\%) y Sevilla (13\%).

La progresiva competencia que en el mercado hortofrutícola mundial se está produciendo, exige cada vez más que las cooperativas de Primer grado se integren en estructuras organizativas comerciales de mayor dimensión, procurando mejorar su capacidad negociadora frente a las grandes transnacionales fruteras y comerciantes internacionales monopolizadores de una importante cuota de mercado. Tal como las empresas se integran vertical y horizontalmente («Holding») para aumentar su rentabilidad, el sector empresarial asociativo debe plantearse estrategias que permitan abaratar costes mediante la concentración de la oferta, ampliación de los calendarios comerciales y variedades ofertadas, como también planificar y adaptar la oferta a la demanda e incentivar la innovación tecnológica entre sus asociados. En este contexto la Cooperativa Surinver, Soc. Coop. se encuentra actualmente integrada en Anecoop, Soc. Coop. Se trata de una cooperativa valenciana de Segundo grado especializada en 
EVOLUCIÓN DE LA COMERCIALIZACIÓN DE LA COOPERATIVA SURINVER (ALICANTE), EN TONELADAS

\begin{tabular}{|c|c|c|c|c|c|c|}
\hline Producto & $\begin{array}{l}\text { 1986-87 } \\
\text { (1) }\end{array}$ & (2) & $\begin{array}{l}\text { 1988-89 } \\
\text { (1) }\end{array}$ & (2) & $\begin{array}{l}\text { 1990-91 } \\
\text { (1) }\end{array}$ & (2) \\
\hline Alcachofa & 977 & 1.151 & 486 & 441 & 431 & 891 \\
\hline Apio & 791 & 4.396 & 1.074 & 4.865 & 591 & 2.892 \\
\hline Bróculi & 45 & 144 & 51 & 31 & 73 & 10 \\
\hline Calabacines & 18 & 100 & 18 & 100 & 19 & 44 \\
\hline Col china & - & 96 & - & - & - & - \\
\hline Coliflor & 3 & 24 & 120 & 57 & 50 & 105 \\
\hline Espárragos & 11 & 14 & - & - & - & - \\
\hline Guisantes & 15 & - & - & - & - & - \\
\hline Habas & 93 & 14 & - & - & - & - \\
\hline Judías & 60 & 118 & 54 & 15 & 23 & 2 \\
\hline Lechugas & 999 & 6.080 & 1.663 & 8.068 & 2.142 & 10.096 \\
\hline Melón & 433 & 81 & 124 & 7 & 95 & - \\
\hline Pimientos & 5.457 & 14.721 & 4.458 & 13.795 & 5.064 & 12.407 \\
\hline Pimiento padrón & - & - & 166 & - & 175 & - \\
\hline Pimiento amarillo & - & - & 14 & 74 & 47 & 158 \\
\hline Pepino & 140 & - & - & - & - & - \\
\hline Sandía & 116 & - & 109 & - & 303 & 508 \\
\hline Tomate & 268 & 82 & 352 & 26 & 43 & - \\
\hline Otros & 23 & - & 58 & 35 & 22 & - \\
\hline Limones & 3.440 & 3.679 & 5.074 & 4.117 & 3.086 & 2.652 \\
\hline Naranjas & 469 & 210 & 79 & 103 & 2.673 & 1.688 \\
\hline Clavel Sim & - & - & 8.148 & 3.856 & 3.914 & 354 \\
\hline Clavel mini & 一 & 一 & 453 & 1.974 & 401 & 92 \\
\hline Clavel Chinera & - & - & 1.840 & 168 & 1.905 & 21 \\
\hline Crisantemos & - & - & 569 & 382 & 1.137 & 164 \\
\hline Gladiolos & - & 一 & 1.955 & 283 & 3.173 & 119 \\
\hline Gerbera & - & 一 & 539 & 180 & 287 & 11 \\
\hline Iris & - & 一 & - & - & 178 & - \\
\hline Paniculata & 一 & 一 & 962 & 230 & 617 & 47 \\
\hline Statice & - & - & 747 & 345 & 294 & 72 \\
\hline Rosa & - & 一 & 483 & 44 & 948 & 115 \\
\hline Otras flores & - & - & 296 & 29 & 433 & - \\
\hline TOTAL FLORES* & 1.794 & 1.391 & 15.992 & 7.491 & 13.287 & 995 \\
\hline $\begin{array}{l}\text { TOTAL HORTALIZAS } \\
\text { Y FRUTAS }\end{array}$ & 15.152 & 32.301 & 13.904 & 31.811 & 14.927 & 31.566 \\
\hline
\end{tabular}

FuENTE: SURINVER, SOC. COOP. Elaboración propia.

Obs.: (1) Mercado Nacional.

(2) Exportación.

(*) Para la campaña 1986-87, en miles de docenas; para las posteriores campañas, en miles de tallos. 
Figura 1. Evolución de la comercialización hortofrutícola. Surinver Soc. Coop. Ltda. FuENTE: Cooperativa Surinver.

la exportación de cítricos y productos hortofrutícolas.

\section{El cooperativismo de segundo grado: Anecoop, Sociedad Cooperativa}

La Agrupación Nacional de Exportación de Cooperativas Citrícolas (Anecoop, Soc. Coop.) nace en el año 1975, encuadrada en la Unión de Cooperativas del Campo (UNA$\mathrm{CO}$ ). Su propósito inicial era representar, a modo de entidad asociativa y sin personalidad jurídica, los intereses cooperativos ante el Comité de Gestión para la exportación de frutos cítricos, ya que en la comercialización externa se dirimían fundamentalmente los beneficios que aportaba la producción de cítricos valencianos. Poco tiempo después se aprueban los Estatutos de funcionamiento (1975) y en octubre del mismo año se inician las actividades comerciales (IMPIVA, 1991, 43).

En el año 1977 son aprobados por la Dirección General de Cooperativas del Ministerio de Trabajo, los estatutos de la Sociedad Cooperativa de Exportación de Frutos Cítricos 
FIGURA 2. Exportación de productos hortofrutícolas de la Cooperativa Surinver hacia los paísses de la CEE en valores porcentuales. Año 1990-1991

(Anecoop, Soc. Coop.), constituyéndose como sociedad cooperativa de Segundo grado, independiente de la Unión Nacional de Cooperativas del Campo.

La constitución de Anecoop en cooperativa de Segundo grado, permitiría la expansión y desarrollo de sus actividades, principalmente comerciales, ya que al agrupar a diferentes cooperativas de Primer grado, además de diversificar la oferta, la concentración de mayores cantidades de productos aumentará la capacidad negociadora en el mercado. En este sentido la cooperativa de Segundo grado ofrece importantes ventajas en relación a aquellas asociaciones cooperativas de Primer grado. El cooperativismo de Primer grado, por su dispersión, en lugar de complementarse entra en competencia, perdiendo gran capacidad negociadora y las posibilidades de mejorar la administración, producción, comercialización y financiación que le proporcionaría el cooperativismo de Segundo y ulterior grado. Esto se traduce en poca capacidad para introducirse en el sistema agroalimentario que funciona en los países de la C.E.E. (MONTERO GARCÍA, A., 1988, 29).

IV.1. Crecimiento social y económico 


\section{CUADRo X}

SURINVER (ALICANTE): EVOLUCIÓN DE LAS EXPORTACIONES HORTOFRUTÍCOLAS, POR PAÍS DE DESTINO (EN TONELADAS)

\begin{tabular}{|lrrrrr|}
\hline País & $\mathbf{1 9 8 6 - 8 7}$ & $\mathbf{1 9 8 7 - 8 8}$ & $\mathbf{1 9 8 8 - 8 9}$ & $\mathbf{1 9 8 9 - 9 0}$ & $\mathbf{1 9 9 0 - 9 1}$ \\
\hline Alemania & 8.157 & 9.347 & 10.336 & 8.967 & 10.032 \\
Bélgica & - & - & - & 155 & - \\
Dinamarca * & 1.273 & 1.233 & 527 & 40 & 100 \\
Francia & 10.290 & 8.189 & 9.337 & 9.213 & 8.903 \\
Holanda & 96 & 267 & 1.564 & 1.041 & 1.617 \\
Italia & 1.838 & 1.114 & 1.163 & 1.154 & 1.783 \\
Portugal & - & - & - & - & 11 \\
R. Unido & 7.367 & 4.424 & 5.499 & 4.546 & 3.747 \\
TOTAL CEE & 29.021 & 24.574 & 28.426 & 25.116 & 26.193 \\
Otros países & 3.280 & 4.317 & 3.385 & 3.985 & 5.373 \\
\hline TOTAL & 32.301 & 28.891 & 31.811 & 29.101 & 31.566 \\
\hline
\end{tabular}

FUENTE: SURINVER, SOC. COOP. Elaboración propia.

Obs.: (*) Hasta la campaña 1989-90 las exportaciones de Dinamarca se incluían junto a las de Suecia y Noruega.

FIgURA 3. Localización de las empresas de carácter asociativo cooperadas en Anecoop Soc.

Coop. Año 1990-1991. 
FIguRa 4. Expansión nacional e internacional de Anecoop Soc. Coop. Año 1990-1991 1, Cooperativas asociadas en España; 2, Filiales en la CEE.

Inicialmente los 31 socios que configuraban esta cooperativa estaban localizados en la Comunidad Valenciana y especialmente en la provincia de Valencia, dedicándose en su inmensa mayoría a la producción y comercialización de cítricos, aunque en la campaña 1976-77 ya se realizaban pequeños envíos de hortalizas. Posteriormente las actividades de esta cooperativa se extienden a las provincias de Alicante y Murcia (vid. figura 3), donde además de los cítricos se empieza a diversificar los envíos con la inclusión de otras frutas (campaña 1977-78).

A diferencia de otras entidades asociativas, la cooperativa Anecoop desde un primer momento no aplicó el principio de exclusividad, esto es cada cooperativa asociada tan sólo asumía el compromiso de aportar el 10 por ciento del total comercializado por la misma. Este principio se corresponde perfectamente con los objetivos de la Cooperativa, ya que en esencia se trata de una entidad puramente económica, encaminada a la consecución de unos fines sociales concretos, para que a través de su estructura cooperativa tenga funcionalidad empresarial competitiva y acorde con el entorno en el que se desenvuelve (MIQUEL, J.; PLANELLS, J. M., 1989, 430).

Sin embargo y como ejemplo de la difícil adaptación del sector agrícola español a fórmulas cooperativas que exigen una rigurosa autodisciplina, en el año 1986 Anecoop 
FIgURA 5. Organigrama funcional anecoop S. Coop.

tuvo que sancionar públicamente a las cooperativas asociadas Sociedad Naranjera Villarealense (Sonavi) de Villareal de los Infantes; Cooperativa Agrícola San Miguel, de Palma de Gandía; Cooperativa de Agrios Simat de Valldigna y la de Nuestra Señora de la Fuente de Villalonga, por incumplir los estatutos que exigen aportar a la cooperativa de Segundo grado, un porcentaje mínimo de su producción (Revista Agricultura y Cooperación, n. ${ }^{\circ}$ 35, 1986).

El éxito alcanzado en la primera etapa (vid. cuadro XI) estimula el ingreso de nuevas cooperativas establecidas en otras Comunidades Autónomas (Andalucía, Extremadura, Castilla-La Mancha y Navarra), lo que induce a ampliar tanto el número de agricultores de base (100.000 en 1988), como los calendarios productivos y comerciales. Asimismo en el 
ANECOOP, SOC. COOP.: EVOLUCIÓN DEL NÚMERO DE SOCIOS Y VOLUMEN COMERCIALIZADO

\begin{tabular}{|rrrr|}
\hline Campaña & $\begin{array}{c}\text { Número } \\
\text { de socios }\end{array}$ & $\begin{array}{c}\text { Volumen } \\
\text { Toneladas }\end{array}$ & $\begin{array}{c}\text { Valor } \\
\text { miles pesetas }\end{array}$ \\
\hline $1975-76$ & 31 & 13.658 & 202.050 \\
$1976-77$ & 34 & 23.021 & 399.924 \\
$1977-78$ & 43 & 28.315 & 731.614 \\
$1978-79$ & 51 & 32.968 & 840.295 \\
$1979-80$ & 57 & 54.690 & 1.529 .042 \\
$1980-81$ & 65 & 61.151 & 1.982 .678 \\
$1981-82$ & 71 & 83.738 & 3.084 .240 \\
$1982-83$ & 75 & 109.420 & 5.223 .315 \\
$1983-84$ & 85 & 144.840 & 6.991 .398 \\
$1984-85$ & 96 & 137.288 & 7.480 .212 \\
$1985-86$ & 109 & 180.101 & 10.738 .150 \\
$1986-87$ & 114 & 209.172 & 12.692 .726 \\
$1987-88$ & 132 & 240.479 & 13.549 .703 \\
$1988-89$ & 127 & 224.706 & 12.582 .010 \\
$1989-90$ & 129 & 196.444 & 1.369 .104 \\
$1990-91$ & 134 & 227.898 & \\
\hline
\end{tabular}

FuENTE: ANECOOP, SOC. COOP. Elaboración propia.

plano internacional la Cooperativa inicia sus actividades comerciales estableciendo sociedades en Francia (Anecoop France), inaugurada en 1979, Reino Unido (Fesa U.K.) en 1985, y Alemania (Anecoop GmbH) en 1985. También en Francia Anecoop France constituye en 1985 una empresa filial (Nadal Fruits) en Perpignan, y monta una oficina comercial en París (vid. figura 4).

La participación en la sociedad anónima Anecoop pre-empaquetados (A.P.S.A.) (1985), Anecoop Tránsitos, S.A. (1988) y Agriconsa, S.A. (1990), completan el amplio espectro de actividades últimamente desarrolladas por esta Cooperativa (vid. figura 5).

\section{IV.2. Estructura comercial y estrategia}

En el año 1990, por volumen total de ingresos monetarios, Anecoop, Soc. Coop. se situaba en el puesto 41 de la clasificación de Empresas alimentarias españolas (vid. cuadro XII). Aunque su génesis está íntimamente asociada a la exportación de cítricos, la demanda en el mercado externo de productos hortícolas y frutas, ha incrementado sustancialmente el abanico de ofertas de Anecoop (vid. cuadro XIII).

Estructuralmente la Cooperativa sufre los mismos problemas que caracterizan al sector hortofrutícola exportador español, es decir extremada atomización de la oferta. En este sentido en la campaña 1989-90 tan sólo existía una cooperativa (Surinver, Soc. Coop.) que contribuía con un volumen superior a 15.000 toneladas. Cooperativas con cantidades comprendidas entre las 5.000 y 15.000 toneladas eran 8 en la misma campaña, que junto a Surinver comercializaban el 39 por ciento del total de Anecoop. 
FACTURACIÓN DE LAS PRINCIPALES EMPRESAS DEL SECTOR AGROALIMENTARIO DE ESPAÑA, AÑO 1990 (EN MILLONES DE PESETAS)

\begin{tabular}{|lc|}
\hline Empresa & Ingresos \\
\hline Nestlé A.E.P.A. & 102.921 \\
Sesostris & 67.000 \\
Mercados en Origen & 64.247 \\
Ebro Cía. Azúcares y Al. & 58.340 \\
Corp. Ibérica Nutrición & 56.177 \\
Nanta S.A. & 45.000 \\
Transáfrica & 44.000 \\
Agropecuaria Guissona & 42.700 \\
Continental Hispánica & 42.000 \\
General Azucarera España & 38.224 \\
Conservera Campofrío & 37.404 \\
Industrias Agrícolas Cía & 35.023 \\
Nutrexpa & 34.000 \\
Transcatalana de Comercio & 31.000 \\
Pescanova & 30.867 \\
Coop. Orensanas & 30.495 \\
Ind. y Comer. Agropecuaria & 29.000 \\
Bimbo & 24.829 \\
Valls Companys & 24.500 \\
Gallina Blanca Purina & 24.100 \\
Industrias Revilla & 23.600 \\
Oscar Mayer & 23.480 \\
Coop. Pro. Avícola Ganadera & 23.476 \\
Kraft General Foods & 22.000 \\
Pascual Hnos & 21.676 \\
Ferruzzi Ibérica & 21.400 \\
Carnes Estellés & 18.500 \\
Coop. Azucarera Acor & 18.400 \\
Fuertes & 18.000 \\
Egra & 18.000 \\
Starlux & 17.418 \\
Freiremar & 17.050 \\
José Anrubia & 16.438 \\
Derivados Lácteos A. & 16.160 \\
L’Agudana & 16.033 \\
Piensos Hens & 15.600 \\
Cerestar Ibérica & 15.287 \\
Luis Suñer & 15.206 \\
Agrop. de Navarra & 14.924 \\
Saprogal & 14.850 \\
Anecoop., Soc. Coop. & 14.602 \\
\hline
\end{tabular}

Fuente: Anuario Fomento de la Producción, 1990. 
ANECOOP, SOC. COOP. EVOLUCIÓN DE LA EXPORTACIÓN HORTOFRUTÍCOLA (EN TONELADAS)

\begin{tabular}{|rrrrr|}
\hline Campaña & Cítricos & Hortalizas & Frutas & TOTAL \\
\hline $1975-76$ & 13.658 & - & - & 13.658 \\
$1976-77$ & 23.021 & - & - & 23.021 \\
$1977-78$ & 27.282 & 204 & 829 & 28.315 \\
$1978-79$ & 29.910 & 794 & 2.264 & 32.968 \\
$1979-80$ & 47.671 & 1.150 & 5.862 & 54.683 \\
$1980-81$ & 50.276 & 3.798 & 7.077 & 61.151 \\
$1981-82$ & 74.123 & 4.739 & 4.876 & 83.738 \\
$1982-83$ & 87.890 & 9.920 & 11.610 & 109.420 \\
$1983-84$ & 116.185 & 15.071 & 13.584 & 144.840 \\
$1984-85$ & 96.106 & 23.808 & 17.374 & 137.288 \\
$1985-86$ & 130.403 & 33.380 & 16.318 & 180.101 \\
$1986-87$ & 152.983 & 30.165 & 26.024 & 209.172 \\
$1987-88$ & 184.836 & 29.174 & 26.469 & 240.479 \\
$1988-89$ & 174.609 & 27.345 & 22.752 & 224.706 \\
$1989-90$ & 161.519 & 20.219 & 14.706 & 196.444 \\
$1990-91$ & 172.276 & 22.692 & 32.930 & 227.898 \\
\hline
\end{tabular}

FuENTE: ANECOOP, SOC. COOP. Elaboración propia.

CUADRo XIV

ANECOOP, SOC. COOP. EVOLUCIÓN DE LAS EXPORTACIONES HORTOFRUTÍCOLAS, POR PAÍSES (EN TONELADAS)

\begin{tabular}{|lrrrrr|}
\hline País & $\mathbf{1 9 8 4 - 8 5}$ & $\mathbf{1 9 8 7 - 8 8}$ & $\mathbf{1 9 8 8 - 8 9}$ & $\mathbf{1 9 8 9 - 9 0}$ & $\mathbf{1 9 9 0 - 9 1}$ \\
\hline Alemania & 69.283 & 104.005 & 107.555 & 76.742 & 97.874 \\
Bélgica & 6.086 & 7.847 & 9.929 & 7.586 & 5.059 \\
Dinamarca & 4.133 & 4.465 & 3.755 & 1.652 & 2.621 \\
Francia & 17.185 & 31.887 & 26.112 & 27.180 & 41.426 \\
Holanda & 5.215 & 9.473 & 11.241 & 9.242 & 8.547 \\
Italia & 1.052 & 7.286 & 4.180 & 1.524 & 2.111 \\
Portugal & - & 395 & 139 & 153 & 178 \\
R. Unido & 10.334 & 8.390 & 11.820 & 14.447 & 11.684 \\
TOTAL CEE & 113.288 & 173.748 & 174.731 & 138.526 & 169.500 \\
Otros países & 24.000 & 66.731 & 49.975 & 57.918 & 54.707 \\
\hline TOTAL & 137.288 & 240.479 & 224.706 & 196.444 & 224.207 \\
\hline
\end{tabular}

FUENTE: ANECOOP, SOC. COOP. Elaboración propia. 
Para la campaña 1990-91 las cooperativas con cantidades comprendidas entre las 5.000 y 15.000 toneladas habían ascendido a 11 y concentraban el 42 por ciento del total comercializado por Anecoop. Sin embargo en el otro extremo se situaban aquellas cooperativas con volúmenes inferiores a las 2.000 toneladas (dimensión extremadamente reducida para este tipo de empresa), que mientras en la campaña 1989-90 representaban el 73 por ciento del total de cooperativas y concentraban el 27 por ciento de la comercialización, en la campaña 1990-91 los porcentajes eran del 72 y 28,5 por ciento respectivamente.

Aunque los países de la Comunidad Económica Europea son el espacio geográfico hacia donde converge la mayor parte de los envíos de Anecoop (vid. cuadro XIV), es importante destacar que mientras en la campaña 1984-85 los países situados fuera del entorno comunitario absorvían el 17 por ciento de las exportaciones, en la campaña 199091 estos países pasaron a importar el 24 por ciento de los envíos de la Cooperativa.

Además de las actividades comerciales externas, en el año 1990 Anecoop comienza a comercializar sus productos en el mercado interno (España). En este sentido la Cooperativa participa con un 20 por ciento del capital de la empresa Hispafruit, constituida por la Confederación de Cooperativas con el único fin de acercar la producción de las cooperativas a los circuitos de distribución internos, eliminando los intermadiarios representados en este caso por los supermercados (grandes superficies). Esta estrategia está íntimamente asociada a la creación del Mercado Único Europeo, que presumiblemente provocará el ingreso en España de un gran número de empresas asociativas y sociedades anónimas de origen comunitario europeo, dispuestas a competir y comercializar sus propias producciones.

\section{Bibliografía}

ÁLVAREZ GÓMEZ, J.: «Las agrupaciones de Productores Agrarios Hortofrutícolas», Revista Estudios Agrosociales, n. ${ }^{\circ}$ 127, Madrid, Ministerio de Agricultura, 1984, pp. 63-97.

COSTA MAS, J. y CANALES, G.: «El cultivo en invernadero y la comercialización agraria en Orihuela y Campo de Cartagena», Cuadernos de Geografía, n. . 27, Valencia, Universidad de Valencia, 1980, pp. 173-202.

GÓMEZ LÓPEZ, J. D.: «El cultivo de invernadero y la agricultura tipo familiar en Europa y España», Actas XI Congreso Nacional de Geografía, Madrid, A.G.E.-Universidad Complutense, 1989, pp. 319-330.

- Los cultivos de invernadero en la fachada del Sureste peninsular, ante el ingreso en la Comunidad Económica Europea. Aspectos socioeconómicos y comerciales. Tesis Doctoral inédita. Universidad de Alicante, Departamento de Geografía Humana, 1992, 3 volúmenes.

GINER GONZÁLEZ, J.: Mutxamel, Mutxamel, Excmo. Ayuntamiento de Mutxamel, 1981, 300 pp. MIQUEL, J. y PLANELLS, J. M.: «Reflexión y autocrítica a la rentabilidad actual de Anecoop, Soc. Coop., en sus aspectos sociales y empresariales», II Jornadas de comercialización y desarrollo de la empresa cooperativa agraria, Alicante, I.C.A.V.- FE.CO.AV., 1989, pp. 429-446.

MONTERO GARCÍA, A.: «Cooperativismo agrario de segundo grado», Revista Agricultura y Comercialización, n. ${ }^{\circ}$ 61, Valencia, 1988, pp. 29-30.

NAVARRO SALA, J. A.: Estudio-análisis de costos y niveles de precios en los mercados de tomate de invierno, Alicante, Servicio comercial de Cosecheros-Exportadores de Tomate de Alicante, 1966, 155 pp.

RODRÍGUEZ BRITO, W.: La agricultura de exportación en Canarias (1940-1980), Santa Cruz de Tenerife, Consejería de Agricultura y Pesca, Gobierno de Canarias, 1986, 571 pp. 